JIRSS (2018)

Vol. 17, No. 02, pp 165-179

DOI: $10.29252 /$ jirss.17.2.8

\title{
Spatial Interpolation Using Copula for non-Gaussian Mod- eling of Rainfall Data
}

\author{
Mehdi Omidi ${ }^{1}$, Mohsen Mohammadzadeh², \\ ${ }^{1}$ Department of Mathematics, Ilam University, Ilam, Iran. \\ ${ }^{2}$ Department of Statistics, Tarbiat Modares University, Tehran, Iran. \\ Received: 04/09/2017, Revision received: 05/02/2018, Published online: 06/08/2018
}

\begin{abstract}
One of the most useful tools for handling multivariate distributions of dependent variables in terms of their marginal distribution is a copula function. The copula families capture a fair amount of attention due to their applicability and flexibility in describing the non-Gaussian spatial dependent data. The particular properties of the spatial copula are rarely seen in all the known copula families. In the present paper, based on the weighted geometric mean of two Max-id copulas family, the spatial copula function is provided. Afterwards, the proposed copula along with the Bees algorithm is used to explore the spatial dependency and to interpolate the rainfall data in Iran's Khuzestan province.
\end{abstract}

Keywords. Spatial copula function, Random field, Max-id copulas.

MSC: 60E05; 62H12.

\section{Introduction}

Identifying the distribution of a random field is required for the analysis of the spatial data. Although the traditional geostatistical predictions have been made by use

Corresponding Author: Mehdi Omidi (Omidi_280@yahoo.com),

Mohsen Mohammadzadeh (mohsen_m@modares.ac.ir). 
of Gaussian random fields, in some settings, this assumption is not realistic and it is necessary to explore the spatial distribution. Copulas are standardized multivariate distributions with uniform marginals that are used to construct multivariate distributions when the distributions of a set of random variables are unknown. The copulas were introduced by Sklar (1959) and profoundly explained by Joe (1997) and Nelsen (2006) as a powerful method for modeling the dependency structure of two or more random variables.

A Gaussian random field copula can be used as a powerful method to build a covariance function for modeling the correlation structure of the spatial location (Omidi and Mohammadzadeh, 2016). However, when the distribution of a random field is unknown, its distribution can be constructed by copulas. Bardossy (2006) utilized noncentral $\chi^{2}$-copula to the analysis of the groundwater quality parameters. His work was extended by Bardossy and Li (2008) and Kazianka and Pilz (2010) for interpolation of continuous random fields. In another study, Graler and Pebesma (2011) introduced the spatial copula function using the weighted arithmetic mean of two copulas. This type of copula is presumed to be applicable for both negative and positive dependency. In this paper according to the weighted geometric mean of two Max-infinitely divisible (Max-id) copulas, another type of spatial copula is provided which only works in positive dependency.

Existence of a great number of produced copulas for each lag is a drawback of this type of spatial copula. This weakness motivates us to use an optimized method for estimating the spatial copula parameters that have a high convergence speed in finding the optimum values.

Bees Algorithm (BA), is a numerical optimization algorithm which is inspired by the behavior of honey bees. BA was introduced and applied by Pham et al. (2005) to optimize continuous functions, then it was developed by Pham and Ghanbarzadeh (2007) for multiple Pareto optimal solutions. Because of its simplicity and ease of implication (Bao and Zeng, 2009), the BA has been widely used in the literature. For example, Pham et al. (2006a) employed it for complex optimization problems, Pham et al. (2006b) coupled the BA and the neural networks for identification of wood defects. Tapkan et al. (2012) and Tapkan et al. (2013) have also resolved their problems using BA fuzzy multi-objective.

The present paper is structured as follows. In Section 2, the copula and dependency properties are briefly reviewed. In Section 3, properties of valid spatial copula functions are presented and a new type of non-Gaussian spatial copula is introduced. In Section 4, the proposed spatial copula, along with modified Bees Algorithm, is employed to explore the spatial dependency and to interpolate the rainfall data of Iran's Khuzestan 
province.

\section{Copula and Some of its Dependency Properties}

The copula functions allow us to analyze the dependency structure of a data set through multivariate distributions. A d-dimensional copula is a multivariate distribution which links multivariate distributions with their univariate marginals. Let $H\left(x_{1}, \ldots, x_{d}\right)$ be a d-dimensional distribution function with the continuous marginal distribution functions $F_{1}, \ldots, F_{d}$. According to Sklar's Theorem (Sklar, 1959), for all $\mathbf{x}=\left(x_{1}, \cdots, x_{d}\right) \in R^{d}$ and $\mathbf{u}=\left(u_{1}, \cdots, u_{d}\right) \in[0,1]^{d}$ where $u_{i}=F_{i}\left(x_{i}\right)$, there exists a unique copula function $C(\cdot)$ such that

$$
H(\mathbf{x})=C(\mathbf{u}) .
$$

Each multivariate copula is bounded by the Frechet-Hoeffding upper and lower bounds as

$$
W(\mathbf{u})=\max \left\{\sum_{i=1}^{d} u_{i}-n+1,0\right\} \leq C(\mathbf{u}) \leq \min \left\{u_{1}, \ldots, u_{d}\right\}=M(\mathbf{u}) .
$$

Note that $W(\cdot)$ is a copula if and only if $d=2$, but $M(\cdot)$ always is a copula and provides maximum dependency among variables. When random variables are independent, their dependency structure is expressed by the product copula $\Pi(\mathbf{u})=\prod_{1=1}^{d} u_{i}$. Here, we consider a number of dependency properties of the random variables that will be expressed in terms of copula functions.

Positively (Negatively) Quadrant Dependency: Continuous random variables $X$ and $Y$ with copula $C(\cdot, \cdot)$, are Positively Quadrant Dependent (PQD) if and only if, $C(u, v) \geq \Pi(u, v)$. They are Negatively Quadrant Dependent (NQD) if, and only if, $C(u, v) \leq \Pi(u, v)$.

Left Tail Decreasing: For a pair $(X, Y)$ of random variables with the copula $C(\cdot, \cdot)$, the random variable $Y$ is said to be Left Tail Decreasing (LTD) in $X$ if, $P(Y \leq$ $y \mid X \leq x)$ is a non-increasing function for all $y$, or equivalently if $C(u, v) / u$ is a non-increasing function of $u$ (Nelsen, 2006).

Totally Positive of order 2: A non-negative function $f(\cdot, \cdot)$ from $\Re^{2}$ to $\Re$ is Totally Positive of order 2 (TP2) if, for all $x_{1} \leq y_{1}$ and $x_{2} \leq y_{2}$,

$$
f\left(x_{1}, x_{2}\right) f\left(y_{1}, y_{2}\right) \geq f\left(x_{1}, y_{2}\right) f\left(y_{1}, x_{2}\right) .
$$


Max-id Copulas: A copula function $C(u, v)$ is said to be Max-id if $C^{r}(u, v)$ is a bivariate distribution function for all $r>0$ (Joe, 1997). Klein et al. (2011) proved that for any two Max-id copulas $C_{1}(u, v)$ and $C_{2}(u, v)$, the function $C_{1}^{\alpha}(u, v) C_{2}^{1-\alpha}(u, v)$ for $\alpha \in[0,1]$ is again a copula.

For any copula function $C(u, v)$, Joe (1997) demonstrated that the following implications are held:

- If $C(\cdot, \cdot)$ is Max-id, then $C(\cdot, \cdot)$ is TP2.

- If $C(\cdot, \cdot)$ is TP2, then $C(\cdot, \cdot)$ is LTD.

- If $C(\cdot, \cdot)$ is LTD, then $C(\cdot, \cdot)$ is PQD.

It can be concluded that if $C(\cdot, \cdot)$ is Max-id, then $C(\cdot, \cdot)$ is $P Q D$.

\section{Spatial Copula Function}

In geostatistics, the distribution of any spatial random field should cover all the lag distances of spatial dependency. Since the correlation between two spatial locations is always positive and it decreases when the distance between locations increases, so the distribution must result in a positive value for the spatial correlation and be a decreasing function for all spatial lags. Based on these properties the copula function $C_{h}(u, v)$ is a valid spatial copula in lag $h$ if it has the following characteristics:

1. $C_{h}(u, v)$ is $P Q D$, because it results in a positive measure of dependency at each lag $h$.

2. $C_{h}(u, v)$ is exchangeable, $C_{h}(u, v)=C_{h}(v, u)$, for any stationary and isotropic random field. Because of stationarity, the joint distribution of the random field at the two locations $s$ and $s+h$ depends only on $h$ and, based on isotropy property, this distribution remains the same in all directions. Accordingly, for any stationary and isotropic random field, the asymmetrical copula is inappropriate to construct a valid spatial copula.

3. $C_{h}(u, v)$ attains the upper Frechet-Hoeffding bound when the lag $h$ tends to zero and it is a product copula when the lag is greater than the range of spatial correlation structure of the data (Kazianka and Pilz, 2010). 
Table 1: The Max-id copulas, their functions along with the value of their parameters.

\begin{tabular}{llc}
\hline Copula & Function & Parameter \\
\hline Clayton $\left(C_{C l}\right)$ & $\left(u^{-\theta}+v^{-\theta}-1\right)^{\frac{-1}{\theta}}$ & $\theta>0$ \\
Ali-Mikhail-Haq $\left(C_{A M H}\right)$ & $\frac{u v}{1-\theta(1-u)(1-v)}$ & $\theta>0$ \\
Gumbel $\left(C_{G}\right)$ & $\exp \left\{-\left[(-\ln u)^{\theta}+(-\ln v)^{\theta}\right]^{\frac{1}{\theta}}\right\}$ & $\theta>1$ \\
Frank $\left(C_{F}\right)$ & $\frac{-1}{\theta} \ln \left[1+\frac{\left(e^{-\theta u}-1\right)\left(e^{-\theta v}-1\right)}{e^{-\theta}-1}\right]$ & $\theta>0$ \\
Joe $\left(C_{J}\right)$ & $1-\left[(1-u)^{\theta}+(1-v)^{\theta}-((1-u)(1-v))^{\theta}\right]^{\frac{1}{\theta}}$ & $\theta>1$ \\
\hline
\end{tabular}

The convex combination of copulas was used to different distances by Graler and Pebesma (2011). Using the weighted geometric mean of two Max-id copulas, we provided another type of spatial copula which depends on the spatial lags and results in a positive measure of dependency. Some Max-id copulas along with the value of their parameters are summarized in Table 1.

Theorem 3.1. Let $C_{h_{i}}(u, v), i=1, \ldots, \ell$ be Max-id copulas and $h_{\ell}$ be the range of spatial correlation of the data, then the function

$$
C_{h}(u, v)=\left\{\begin{array}{cl}
M^{\lambda_{1}}(u, v) C_{h_{1}}^{1-\lambda_{1}}(u, v) & 0 \leq h \leq h_{1} \\
\vdots & \vdots \\
C_{h_{i-1}}^{\lambda_{i}}(u, v) C_{h_{i}}^{1-\lambda_{i}}(u, v) & h_{i-1} \leq h \leq h_{i} \\
\vdots & \vdots \\
C_{h_{\ell}}^{\lambda_{\ell}}(u, v) \Pi^{1-\lambda_{\ell}}(u, v) & h_{\ell-1} \leq h \leq h_{\ell} \\
\Pi(u, v) & h>h_{\ell}
\end{array}\right.
$$

is a valid spatial copula, where $\lambda_{i}=\frac{h_{i}-h}{h_{i}-h_{i-1}}, i=1, \ldots, \ell$.

Proof. It is straightforward to illustrate Equation (3.1) attains $M(u, v)$ for distances near to zero and $\Pi(u, v)$ for distances greater than the range of spatial correlation of data. Since the copula $C_{h}(u, v)$ is Max-id, $C_{h}(u, v)$ is PQD and it results in positive measure of dependency in lag $h$ (Omidi and Mohammadzadeh, 2014).

In order to measure the spatial dependency, Spearman correlation in terms of the copula (Nelson, 2006) can be used for each distance lag in Equation (3.1) by

$$
\widetilde{\rho}_{s}\left(h_{i}\right)=12 \int_{0}^{1} \int_{0}^{1} C_{h_{i-1}}^{\lambda_{i}}(u, v) C_{h_{i}}^{1-\lambda_{i}}(u, v) d u d v-3, \quad i=1, \ldots, \ell-1 .
$$

where $\widetilde{\rho}_{s}\left(h_{i}\right)$ is Spearman correlation in the i-th lag. 

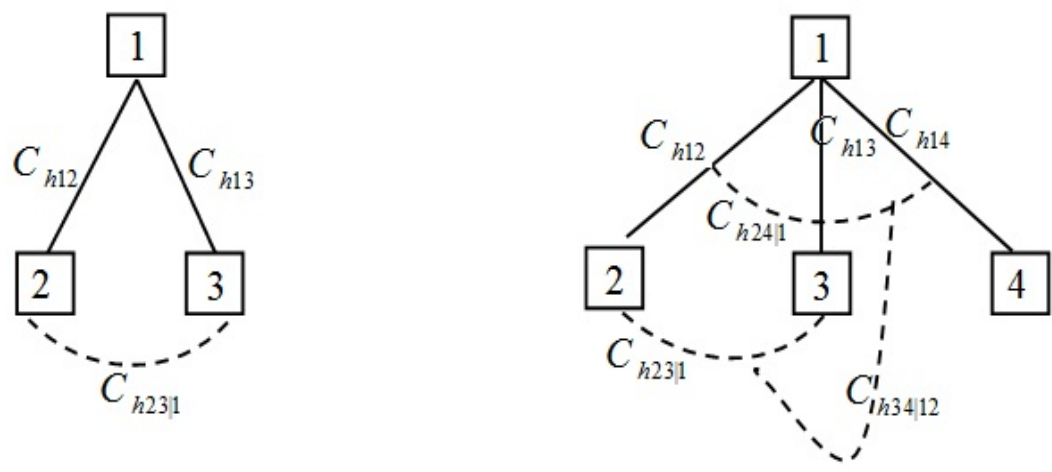

Figure 1: Graphical C-vine on three and four variables with spatial and conditional spatial copulas.

The spatial copula in Equation (3.1) provides a bivariate copula in each lag. In order to construct a multivariate spatial copula, the pair copula can be used where the multivariate density is obtained by the product of all bivariate copula densities in terms of a regular vine. In this setting, Bedford and Cooke (2002) and Aas et al. (2009) investigated two main types of pair copula containing D-vine and a canonical vine. Our approach will be based on the canonical vine in which one particular variable is concentrated and the decomposition is built upon it. The canonical form of the pair copula (C-vine) on three and four variables with spatial and conditional spatial copulas is depicted in Figure 1. Based on this figure, the density function for 3 variables $X\left(s_{1}\right)$, $X\left(s_{2}\right)$ and $X\left(s_{3}\right)$ can be written as

$$
\begin{aligned}
f\left(x\left(s_{1}\right), x\left(s_{2}\right), x\left(s_{3}\right)\right) & =f_{1}\left(x\left(s_{1}\right)\right) f_{2}\left(x\left(s_{2}\right)\right) f_{3}\left(x\left(s_{3}\right)\right) \\
& \times c_{h_{12}}\left(F_{1}\left(x\left(s_{1}\right)\right), F_{2}\left(x\left(s_{2}\right)\right)\right) \\
& \times c_{h_{13}}\left(F_{1}\left(x\left(s_{1}\right)\right), F_{3}\left(x\left(s_{3}\right)\right)\right) \\
& \times \quad c_{h_{23 \mid 1}}\left(F\left(x\left(s_{2}\right) \mid x\left(s_{1}\right)\right), F\left(x\left(s_{3}\right) \mid x\left(s_{1}\right)\right)\right),
\end{aligned}
$$

where $f_{i}\left(x\left(s_{i}\right)\right)$ is the marginal density function of $X\left(s_{i}\right)$ and $c_{h_{i}}(u, v)=\frac{\partial^{2} C_{h_{i}}(u, v)}{\partial u \partial v}$ is the copula density and, finally,

$$
F\left(x(i) \mid x\left(s_{1}\right)\right)=\left.\frac{\partial C_{h_{i 1}}(u, v)}{\partial v}\right|_{u=F\left(x\left(s_{i}\right)\right), v=F\left(x\left(s_{1}\right)\right)} .
$$


Generally, the n-dimensional density function for a canonical vine can be obtained by the edges of the vine and a product of the marginal densities which is given by

$$
\begin{aligned}
f_{1, \ldots, n} & =\prod_{k=1}^{n} f_{k}\left(x\left(s_{k}\right)\right) \\
& \times \prod_{i=1}^{n-1} \prod_{j=i+1}^{n} c_{h_{i j \mid i+1, \cdots, j-1}}\left(\left(F_{i} \mid i+1 \cdots j-1\right),\left(F_{j} \mid i+1, \cdots, j-1\right)\right) .
\end{aligned}
$$

where the conditional distribution can be achieved by

$$
F_{i \mid v \bigcup j}\left(x_{i} \mid x_{v} \bigcup j\right)=\left.\frac{\partial C_{h_{i j \mid v}}\left(u_{i}, u_{j}\right)}{\partial u_{j}}\right|_{u_{i}=F_{i \mid v}\left(x_{i} \mid x_{v}\right), u_{j}=F_{j \mid v}\left(x_{j} \mid x_{v}\right)} .
$$

The prediction for unobserved locations can be obtained by using Equation (3.2) and Equation (3.3) and calculating the median of conditional copula (Bardossy and Li, 2008) in

$$
\hat{x}_{\text {predicted }}=F_{X}^{-1}\left(x=C^{-1}\left(0.5 \mid U_{1}=u_{1}, \ldots, U_{n}=u_{n}\right)\right) .
$$

\section{Bees Algorithm}

Having a great number of generated copulas for all distances are a drawback of Equation (3.1), for example by choosing $k$ different copula families, $2 k$ copulas in each one of the distances lag $h_{1}$ and $h_{\ell-1}$, and for other distances, $k(k-1)$ different copulas must be fitted. This weakness demands the use a strong algorithm which decreases the computational convergence time and retains high accuracy for estimation of copula parameters.

Bees Algorithm (BA) is a numerical optimization method which has been created based on the behavior of honey bees. Technically, in BA the first value of parameters (first population) are randomly selected in the space of parameters. Afterwards, new values are generated by the mechanism of the neighborhood and random search. The basic code for BA for minimizing of -log-likelihood function can be summarized as the following steps (Phame et al., 2006a):

Step 1: Generating $n$ random numbers on the space of copula parameters and evaluating the -log of the likelihood function.

Step 2: Start While (while stopping criterion does not meet): 
Step 3: Form the new population.

Step 4: Those bees that have the minimum value of -log-likelihood function are chosen. $m$ visited sites by them are selected for neighborhood search.

Step 5: Recruiting bees for the selected sites (more bees for the sites which have less value of -log-likelihood function), then evaluating and sorting the new values of -log-likelihood function in a decreasing order.

Step 6: Selecting the best bees which have the minimum value of -log-likelihood function from each patch.

Step 7: Finding new potential solutions based on a random search for the other bees. $n-m$ random numbers are generated on the space of copula parameters and evaluating the -log-likelihood function.

Step 8: End While.

Based on the above steps, running BA requires to have the number of populations $(n)$, the number of sites $(m)$, the number of elites $(e)$, the number of bees for a neighborhood search of elites, and the number of bees for a neighborhood search around the other sites.

\section{$5 \quad$ Application}

The south west of Iran, where runoffs enter into the Khozestan basin, provides the focus of this paper. The region includes 137 rainfall stations (Figure 2). The range of spatial dependency was identified by fitting exponential, Gaussian and spherical models to the empirical variogram. Due to the presence of outliers, the robust empirical estimator (Cressie and Hawkins, 1980) was employed. Based on the Residual Sum of Squares (RSS) of variogram estimates, which gives 390.7099, 390.7099 and 248.357 for exponential, Gaussian and spherical models, respectively, the spherical model was chosen. As indicated in Figure 3, the sill parameter is approximately 31.650, nugget effect is 1.771 and the range distance is $73.716 \mathrm{Km}$. To check the isotropy, Figure 4 refers to the empirical variogram in directions 0, 30, 60, 90, 120 and 150 degree. Spatial arrangement of aforementioned directions in the range of spatial dependency suggests its proximity with the isotropic pattern of correlation structure of the data, which shows that the exchangeable copulas must be fitted to the data.

The small lag distances cause the sample size drastically decrease, then it affects on the accuracy of fitting the copula functions. So the lag distances were considered by 15 


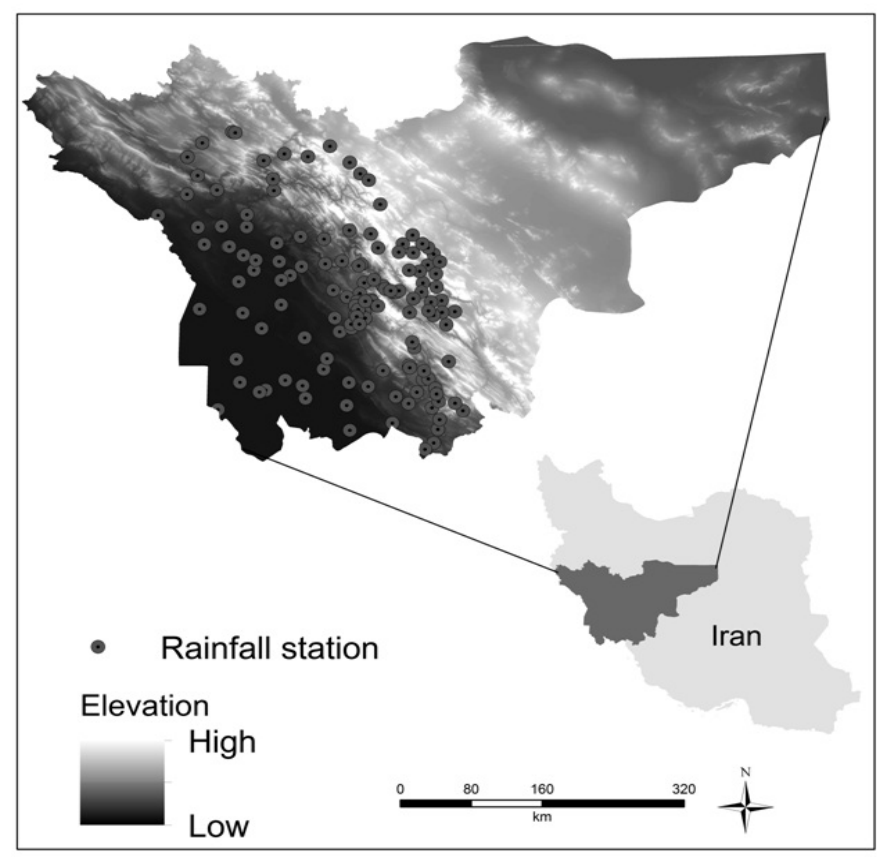

Figure 2: Map and locations of the rainfall monitoring stations.

$\mathrm{km}$. Therefore, the range of spatial dependency was divided into five spatial dependency lags plus one lag to show the independent dependency for the distances greater than the range. The most common distribution to fit rainfall data is Gamma and Lognormal (Cho et al., 2004), in which the Kolmogorov-Smirnov test shows the Gamma distribution with p_value $=0.487$, the shape parameter $\alpha=4.641$, and the scale parameter $\beta=114.540$ fits to the data. To construct a multivariate random field distribution, the Max-id copulas in Table 1 were employed on the first five lags, accordingly 80 weighted geometric copulas were fitted, in which the marginal distribution of the copula is attained based on cumulative Gamma distribution. The $\mathrm{R}$ software, version 3.4.1 has been used for analysis and making the geostatistical plots.

Using more functions results in arising more uncertainty in the estimation of the spatial copula parameters. To overcome this issue, the Bees Algorithm was used. Parameters were estimated by minimizing $-\log L_{c_{h}}(\boldsymbol{\theta} \mid \mathbf{u}, \mathbf{v})$, where $L_{c_{h}}(\cdot)$ refers to the likelihood function provided by the copula density $c_{h}$ and $\boldsymbol{\theta}$ is the copula parameters vector. Table 2 contains the BA features and their considered values. 


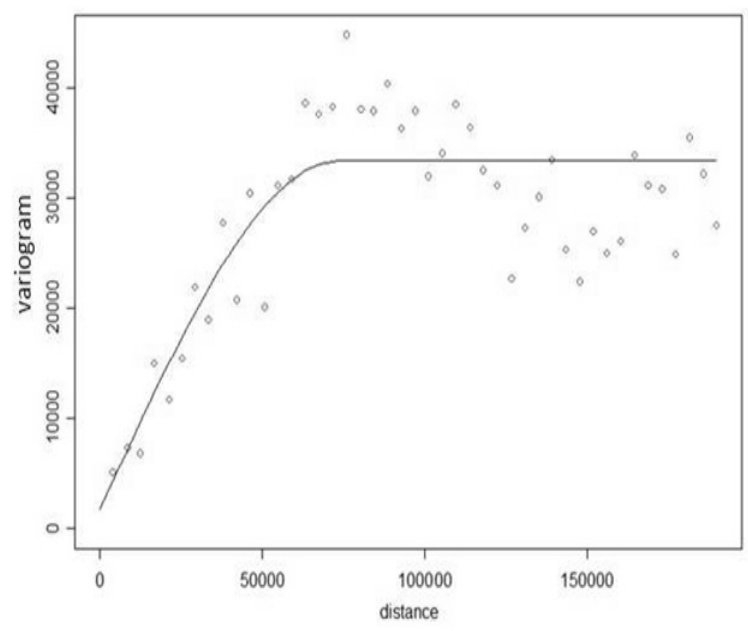

Figure 3: Fitted spherical model to the robust empirical variogram.

Table 2: Features of the BA with their corresponding values.

\begin{tabular}{clc}
\hline Features & Description & Values \\
\hline$n$ & number of populations & 120 \\
$m$ & number of selection best populations & 60 \\
$e$ & number of elites & 20 \\
$n_{e p}$ & number of bees for neighborhood around elites & 12 \\
$n_{s p}$ & number of bees for neighborhood search around other sites & 6 \\
\hline
\end{tabular}

To ensure the accuracy and precision of the parameters estimation and to control the effect of random selection, the minimum values of $-\log L_{c_{h}}(\boldsymbol{\theta} \mid \mathbf{u}, \mathbf{v})$ are considered as the best values and attained by repeating the BA in 10 independent runs.

For each lag, the estimation of parameters was considered, when the plot of the mean and the minimum of the best values in 10 runs were converged and approximately coincided after a certain iteration. The best copula in each lag was chosen based on the minimum value of Akaike Information Criterion $A I C=-2 \log L_{c_{h}}(\boldsymbol{\theta} \mid \mathbf{u}, \mathbf{v})+2 p$, where $p$ is the number of model parameters. Table 3 presents the best copula functions, parameters estimation and their corresponding AICs, in which $\lambda$ was obtained by the median distance of pairs falling within the same interval. Matlab 2014b has been used to run the BA and to estimate the copula parameters.

Figure 5 shows the convergence plots of the mean and the minimum of the best values in the first four lags. The plots show that in lags 1, after 10 iterations, and for the others, after 25 iterations of BA, the plots will converge. The convergence plot and 


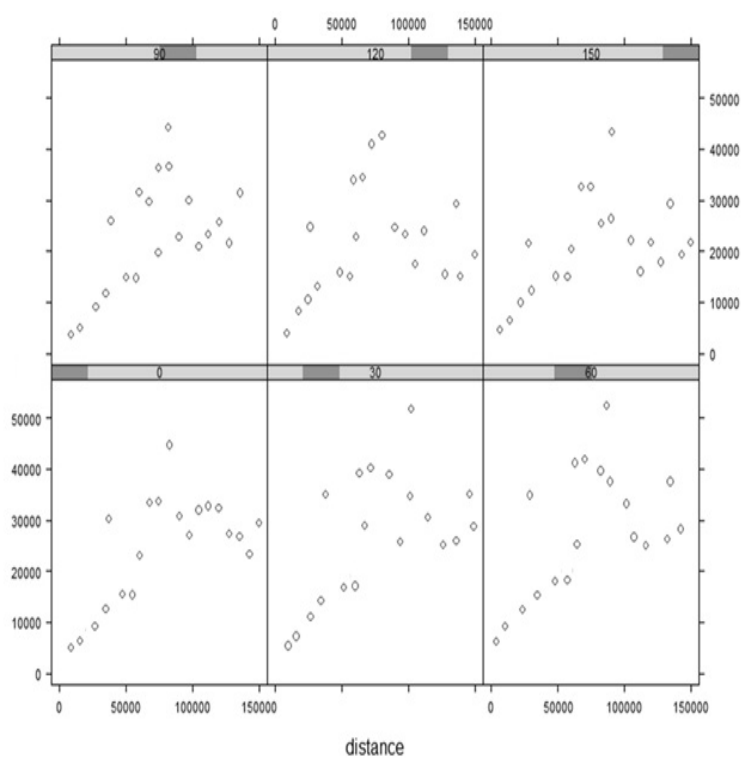

Figure 4: Empirical variogram plots in different directions.

its results in lag 5 are similar to lag 1.

Table 3: The selected copula, estimated parameters with their corresponding AICs in each lag.

\begin{tabular}{lcllcl}
\hline Lag & $\lambda$ & Best Copula & $\theta_{1}$ & $\theta_{2}$ & AIC \\
\hline $0-15$ & 0.7010 & $C_{F}^{\lambda_{1}}\left(u, v, \theta_{1}\right) M^{1-\lambda_{1}}(u, v)$ & 11.7460 & - & -80.6514 \\
$15-30$ & 0.5608 & $C_{C_{2}^{l}}^{\lambda_{2}}\left(u, v, \theta_{1}\right) C_{F}^{1-\lambda_{2}}\left(u, v, \theta_{2}\right)$ & 2.9714 & 8.0659 & -181.2144 \\
$30-45$ & 0.5412 & $C_{C_{3}^{l}}^{\lambda_{4}}\left(u, v, \theta_{1}\right) C_{F}^{1-\lambda_{3}}\left(u, v, \theta_{2}\right)$ & 1.8033 & 4.4770 & -161.0079 \\
$45-60$ & 0.4995 & $C_{C_{4}^{l}}^{\lambda_{1}}\left(u, v, \theta_{1}\right) C_{G}^{1-\lambda_{4}}\left(u, v, \theta_{2}\right)$ & 1.4427 & 1.7044 & -169.9817 \\
$60-73.72$ & 0.6017 & $C_{C l}^{\lambda_{5}}\left(u, v, \theta_{1}\right) \Pi^{1-\lambda_{5}}(u, v)$ & 2.0024 & - & -99.0741 \\
\hline
\end{tabular}

Table 4 contains Spearman correlations for raw data shown by $\hat{\rho}_{s}(h)$ and $\widetilde{\rho}_{s}(h)$ in each spatial distance lag. Both estimations show the decreasing measures of the spatial dependency when the lags are increased to the range. In order to conduct the interpolation, the multivariate copula was obtained by connecting each observation to the four nearest neighbors. This process was done for 20 randomly selected points. The predicted value of these points was obtained by replacing the general form of fivedimensional C-vine ( $n=5$ in Equation (3.2)) and Equation (3.3) in Equation (3.4). Moreover, the relative efficiency of our model was obtained as 5.3907 in terms of root 

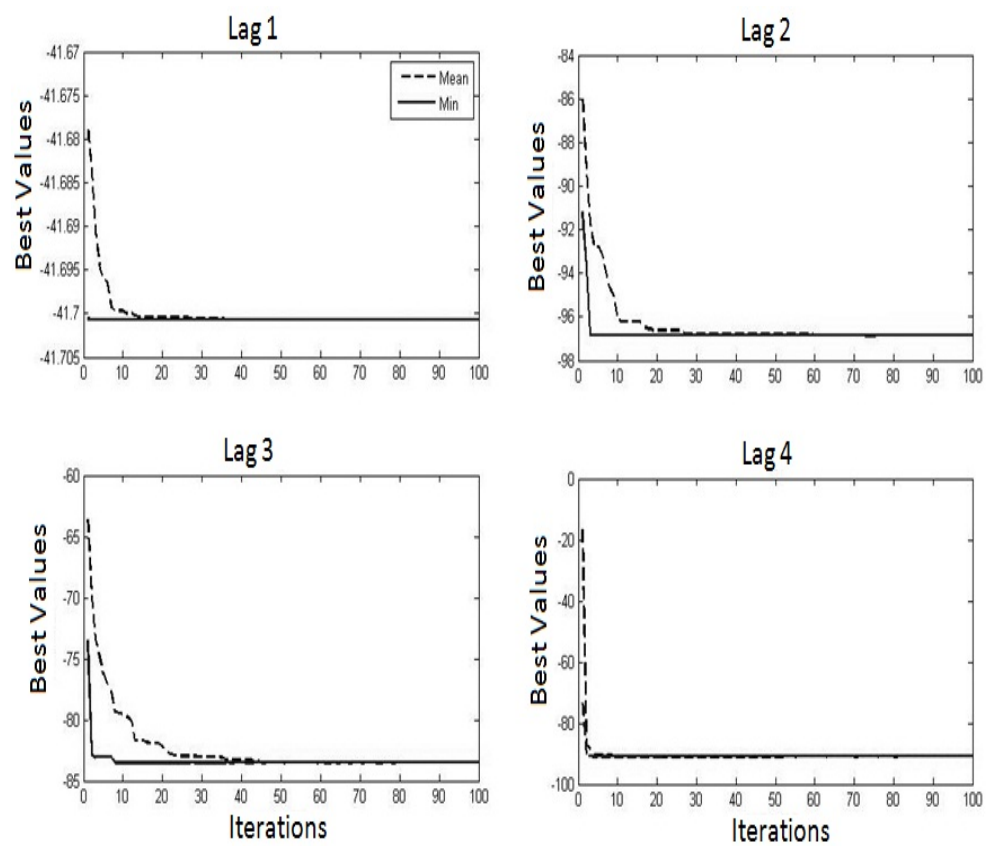

Figure 5: Convergence plots of the mean and the minimum values for the first four lags.

mean square deviation (RMSD), where the prediction map for these points is shown in Figure 6. Also, for assessing our model, the achieved RMSD based on Equation (3.1) is compared with the model constructed by Graler and Pebesma (2011) with $R M S D=5.6775$, which shows that the model based on Equation (3.1) is relatively better than any other model for the data of runoffs enter into the Khuzestan basin.

\section{Conclusion}

In the present paper, we introduced a new type of spatial copula via the integration of two Max-id copula functions which can be used for construction of the distribution of non-Gaussian random field. It was revealed that the developed model always produces positive dependency. Then, to estimate the model parameters, the Bees Algorithm (BA) was used. The accuracy of BA was assessed by plotting the mean and minimum diagram of the best values in 10 independent runs. While the pair copula is capable of constructing the multivariate spatial distribution, it needs intensive calculation for 
Table 4: Spearman correlation for the data and the best copula in each lag.

\begin{tabular}{cccccc}
\hline Correlation & Lag 1 & Lag 2 & Lag 3 & Lag 4 & Lag 5 \\
\hline$\hat{\rho}_{s}(h)$ & 0.7108 & 0.5568 & 0.4705 & 0.4347 & 0.2386 \\
$\widetilde{\rho}_{s}(h)$ & 0.9398 & 0.8051 & 0.6201 & 0.5139 & 0.2919 \\
\hline
\end{tabular}

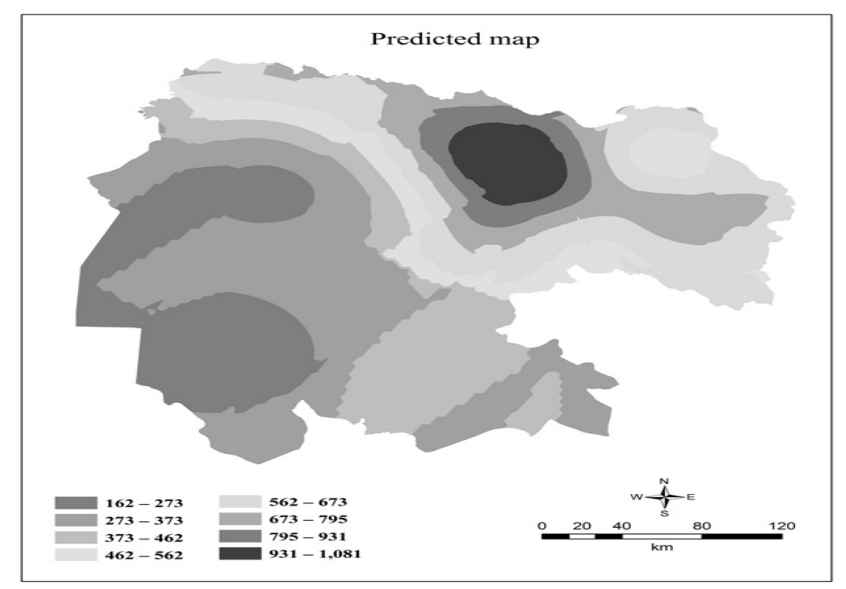

Figure 6: The prediction map for runoffs enter into the Khuzestan basin based on Equation. (3.1)

interpolation when the number of neighbors increases. Although the weighted geometric mean was used to build the spatial copula function, the alternative methods such as C-vine composite likelihood (S-LCVCL) applied by Erhardt et al. (2015a and 2015b) can be used for spatial interpolation.

\section{Acknowledgement}

The authors are thankful to the referees for their many helpful comments that greatly improved this paper. We also wish to acknowledge for the support from the Center of Excellence for Spatial Data Analysis in Tarbiat Modares University.

\section{References}

Aas, K., Czado, C., Frigessi, A. and Bakken, H. (2009), Pair-copula constructions of multiple dependence. Insurance Mathematics Economics, 44(2), 182198. 
Bao, L. and Zeng, J. C. (2009), Comparison and analysis of the selection mechanism in the artificial bee colony algorithm. Ninth International Conference on Hybrid Intelligent Systems.

Bardossy, A. (2006), Copula-based geostatistical models for groundwater quality parameters. Water Resources Research, 42, W11416.

Bardossy, A. and Li, J. (2008), Geostatistical interpolation using copulas. Water Resources Research, 44, W07412.

Cherubini, U., Luciano, E. and Vecchiato, W. (2004), Copula Methods in Finance. John Wiley and Sons.

Bedford, T. and Cooke, R. M. (2002), Vines- A new graphical model for dependent random variables. Annals of Statistics, 30, 1031-1068.

Cho, H. K., Bowman, K. P. and North, G. R. ( 2004), A Comparison of gamma and lognormal distributions for characterizing satellite rain rates from the tropical rainfall measuring mission. Journal of Applied meteorology, 43, 1586-1597.

Cressie, N. A. C. and Hawkins, D. M. (1980), Robust estimation of the variogram. Journal of the International Association for Mathematical Geology, 12, 115-125.

Erhardt, T. M., Czado, C. and Schepsmeier, U. (2015a), Spatial composite llikelihood inference using local C-vines. Journal of Multivariate Analysis, 138, 74-88.

Erhardt, T. M., Czado, C. and Schepsmeier, U. (2015b), R-vine models for spatial time series with an application to daily mean temperature. Biometrics, 71(2), 323-332.

Graler, B. and Pebesma, E. (2011), The pair-copula construction for spatial data: a new approach to model spatial dependency. Spatial Statistics, 7, 206-211.

Joe, H. (1996), Families of m-variate distributions with given margins and $m(m-1) / 2$ bivariate dependence parameters, In: L. ruschendorf and B. Schweizer and M., D., Taylor (ed.). Distributions with fixed marginals and related topics, 120-141.

Joe, H. (1997), Multivariate Models and Dependence Concepts. Chapman and Hall, New York.

Kazianka, H. and Pilz, J. (2010), Spatial interpolation using copula-based geostatistical models. In: Atkinson, P., Lloyd, C.(Eds.), Geo ENVVII-geostatistics for environmental applications. Springer, Berlin, 307-320. 
Klein, I., Matthias, F. and Thomas, P. (2011), Weighted power mean copulas: theory and application. FAU Discussion Papers in Economics 01/2011, Friedrich-Alexander University Erlangen-Nuremberg, Institute for Economics.

Nelsen, R. B. (2006), An Introduction to Copulas. Second Edition, Springer.

Omidi, M. and Mohammadzadeh, M. (2014), Determination of structural dependence of spatial data with copula functions. Journal of Science Kharazmi University, 13(3), 767-778 (In Persian).

Omidi, M. and Mohammadzadeh, M. (2016), A New Method to Build Spatio-Temporal Covariance Functions: Analysis of Ozone Data. Statistical Papers, 57(3), 689-703.

Pham, D. T., Ghanbarzadeh, A., Koc, E., Otri, S., Rahim, S. and Zaidi, M. (2005), The Bees Algorithm. Technical Note, Manufacturing Engineering Center, Cardiff University, UK.

Pham, D. T., Ghanbarzadeh, A., Koc, E., Otri, S. and Zaidi, M. (2006a), The Bees Algorithm- A novel tool for complex optimization problems. Proceedings of the 2nd Virtual International Conference on Intelligent Production Machines and Systems (IPROMS 2006), Elsevier, Cardiff, 454-459.

Pham, D. T., Soroka, A. J., Ghanbarzadeh, A., Kog, E., Otri, S. and Packianather, M. (2006 b), Optimising neural networks for identification of wood defects using the bees algorithm. IEEE International Conference on Industrial Informatics, 8, 1346-1351.

Pham, D. T. and Ghanbarzadeh, A. (2007), Multi-Objective optimisation using the bees algorithm. Proceedings of IPROMS 2007 Conference.

Sklar, A. (1959), Fonctions de repartition a n dimensions et leurs marges. Publications de l'Institut de Statistique de l'Universite de Paris, 8, 229-231.

Tapkan, P., Ozbakir, L. and Baykasoglu, A. (2012), Bees algorithm for constrained fuzzy multi-objective two-sided assembly line balancing problem. Optimization Letters, 6(6), 1039-1049.

Tapkan, P., Ozbakir, L. and Baykasoglu, A. (2013), Solving fuzzy multiple objective generalized assignment problems directly via bees algorithm and fuzzy ranking. Expert Systems with Applications, 40(3), 892-898. 
